\title{
MIPAS Level 2 Processor Prototype: from validation to operation
}

\author{
MARC BERNAU1, ${ }^{*}$, HeIDRUN WEBER ${ }^{2}$, MichaEl SCHMitT ${ }^{1}$, \\ SVEN BARTHA ${ }^{1}$, AND ROLAND GESSNER ${ }^{1}$ \\ ${ }^{1}$ Astrium GmbH \\ \{marc.bernau | michael.schmitt | sven.bartha | roland.gessner\} (at)astrium.eads.net \\ 2 ESA ESTEC \\ heidrun.weber(at)esa.int
}

\section{INTRODUCTION}

$\coprod^{\mathrm{n}}$ $\mathrm{n}$ the research field of atmospheric chemistry a central question for acquired data sets is about validation. Have the data been validated to be useful for science? Has the data set been compared to other data sets? If deviations occur, which cause could be identified? Ultimately, two causes are possible when the same scene is observed: either the acquired raw data set is erroneous (hardware problem) or the data processing infers erroneous information (software problem). In order to make sure that the software works as expected, software validation plays a key role in the overall data set validation campaigns. This paper deals with operational software validation, which is an important component of the entire scientific validation chain.

In the framework of the Michelson Interferometer for Passive Atmospheric Sounding (MIPAS) mission, a sophisticated iterative algorithm scheme has been developed by many contributors, which can be used to infer the concentration of atmospheric trace gases from the measured spectra. Actually, this algorithm has been implemented in three instances for the operational phase of the mission with each fulfilling dedicated tasks:
- a scientific core implementation for proof of feasibility and scientific validation named Optimized Retrieval Model (ORM) coded according to an Algorithm Theoretical Baseline Document (ATBD) [Carli et al., 2013],

- an industry standard implementation of the scientific algorithm including the framework around this algorithm for performance reference and operational processor validation alias MIPAS Level 2 Processor Prototype (ML2PP), and

- a ground segment implementation for operational processing mirroring the ML2PP algorithm referred to as Instrument Processing Facility (IPF).

In this paper, Astrium's ML2PP used for processing calibrated MIPAS spectra (included in the level 1B product) into atmospheric profiles (included in the level 2 product) is presented in terms of its

- General processing scheme,

- Role during the mission,

- Software validation concept.

\footnotetext{
* Corresponding author: Marc Bernau

Dept. System Performance Verification \& User Ground Segments, Claude-Dornier-Strasse, 88039 Friedrichshafen, Germany
} 


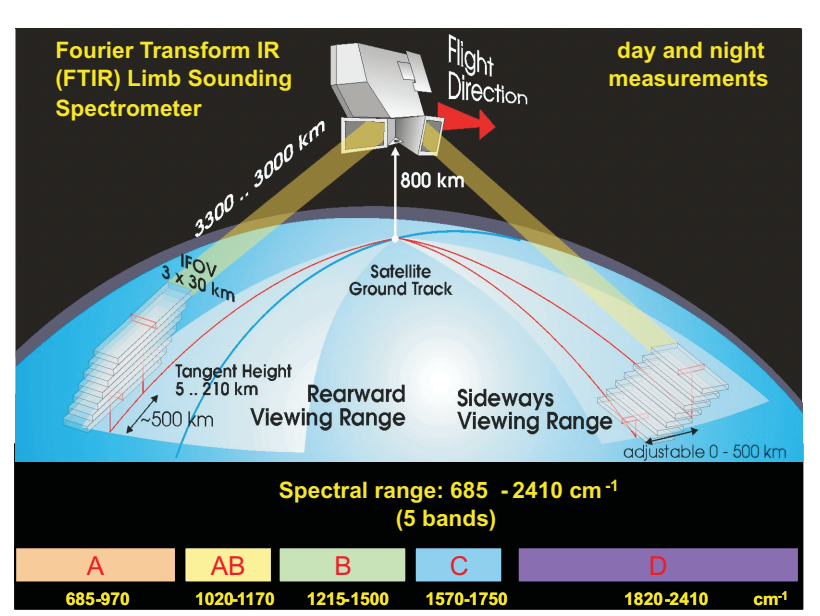

Figure 1: MIPAS measurement scheme

The outline of this paper is as follows. In section 2, a brief introduction of the MIPAS instrument aboard ENVISAT, which was operational from 2002 until 2012, is given. Then, the processing scheme from level 1B to level 2 is briefly described in section 3 . Section 4 recalls the history of the algorithm development, and section 5 presents the software validation concept. Section 6 concludes this paper.

\section{THE MIPAS MISSION}

MIPAS, being one of three atmospheric chemistry instruments aboard ESA's largest Earth Observation satellite ENVISAT, is a limb-sounding FTIR (Fourier transform infrared) spectrometer observing atmospheric data in the mid-infrared range from $4.15 \mu \mathrm{m}$ to $14.6 \mu \mathrm{m}$ with high spectral resolution. The measured data set is very rich in information as each spectrum comprises of the order of 50000 spectral samples.

MIPAS was designed to detect limb emission spectra in the Earth's lower, middle and upper atmosphere (cf. Figure 1), covering tangent heights from 5 to $150 \mathrm{~km}$. During the operational phase, MIPAS gathered spectrally high resolved data in five spectral bands, which can be used for absorption spectroscopy. After in-flight anomalies of the MIPAS moving mirror, starting in March 2004, the instrument operation had to be suspended.

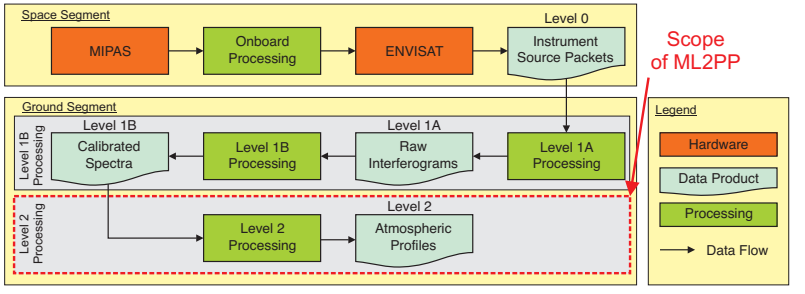

Figure 2: MIPAS data processing

After an analysis period, however, MIPAS could be recovered starting from a 35\% duty cycle in January 2005 to a full mission with an optimized spectral resolution (see also [Raspollini et al., 2013]). The MIPAS in-flight operational performance stabilized gradually, until the observed anomaly pattern that led to the mission interruption had practically disappeared. As a result of the modified spectral resolution, also the ground processing algorithms needed to be adapted accordingly.

MIPAS was operated in two viewing ranges, a rearward viewing range (in anti-flight direction of the satellite) and sideways. Further, instrument calibrations were used for regular radiometric calibrations (using offset calibrations and measurements from an internal blackbody) and line-of-sight calibrations to correct for pointing errors. For further readings about the mission and the MIPAS instrument refer to [Raspollini et al., 2013].

\section{PROCESSING}

The MIPAS data processing takes place sequentially as presented in Figure 2. The level 2 processing forms the final part of a processing chain, taking as input the level 1B (L1B) product, which contains calibrated spectra. It outputs atmospheric profiles of pressure, temperature and dedicated trace gases as a function of altitude. The number of different trace gases in the retrieval scheme has increased from initially six species for the first ML2PP version $\left(\mathrm{O}_{3}\right.$, $\mathrm{H}_{2} \mathrm{O}, \mathrm{HNO}_{3}, \mathrm{CH}_{4}, \mathrm{~N}_{2} \mathrm{O}, \mathrm{NO}_{2}$ ) to ten species for version 6.00 (plus: F11, F12, $\mathrm{N}_{2} \mathrm{O}_{5}, \mathrm{ClONO}_{2}$ ) and to 15 species for ML2PP version 7.00 (plus: F22, F14, $\left.\mathrm{COF}_{2}, \mathrm{CCl}_{4}, \mathrm{HCN}\right)$. The recently added species 
show weaker spectral signatures either due to low atmospheric volume mixing ratio or low emission/absorption per molecule or both. The capability to retrieve such weak species demonstrates the quality of the Level 2 processing algorithm scheme and requires high accuracy of the implementation. The general processing approach is schematically shown in Figure 3. Besides the L1B product, several auxiliary files (pointing information, spectral data, cross-section data, initial guess data, and program settings) are required for the level 2 processing. Optionally, also European Centre for Medium-Range Weather Forecasts (ECMWF) files can be provided to the ML2PP.

The level 2 algorithm works in a scan by scan fashion, where one scan consists of a set of calibrated spectra at different tangent altitudes (sweeps). For each scan at first the pressure and temperature profiles $(\mathrm{p}, \mathrm{T})$ are retrieved from $\mathrm{CO}_{2}$ observations assuming a fixed atmospheric $\mathrm{CO}_{2}$ profile. With the retrieved pressure and temperature profiles then kept fixed, the different trace gas retrievals are performed one after the other. The initial guess is the starting point for each of the retrievals, and plays a crucial role for its convergence. Depending on availability, the initial guess is an optimized composition of climatological profiles, ECMWF profiles (for $\mathrm{p}, \mathrm{T}$ retrieval only), or retrieved profiles from the same or the previous scan.

The retrieval itself is based on a LevenbergMarquardt algorithm, an iterative scheme that combines the advantages of the Newton-Gauss algorithm and the method of gradient descent for finding the minimum of a specific function. In the case of the MIPAS L2 processing, the function to be minimized is the difference between the measured spectrum and a simulated spectrum that is produced by the current set of atmospheric profiles in the iteration. If the defined convergence criteria are fulfilled, the retrieval is successfully terminated. Otherwise, a new set of atmospheric profiles is determined based on the difference of the spectra of the previous itera-

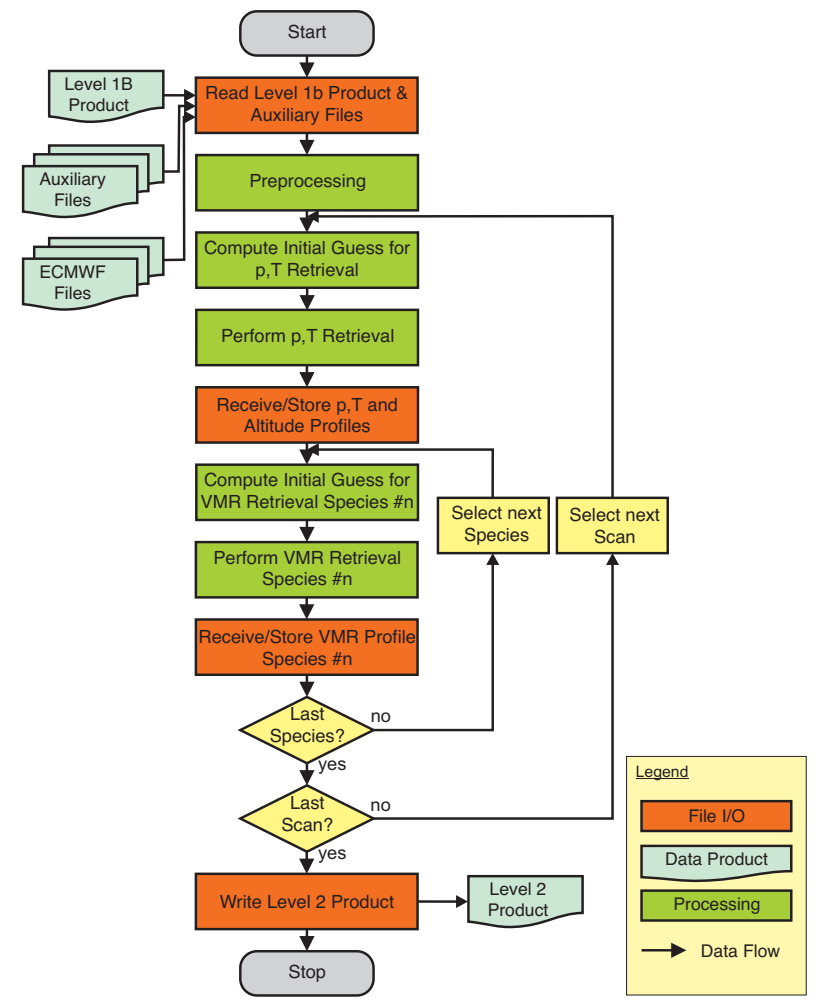

Figure 3: Level 2 processing algorithm

tion. For detailed information on the algorithm refer to [Carli et al., 2013].

\section{LEVEL 2 ALGORITHM DEVELOPMENT}

In section 1, three level 2 processors have been introduced. The dedicated purposes and their role during the mission are summarized in this section.

\section{ORM}

The scientific processor to be used as scientific reference was determined in a shoot-out of several approaches to the level 2 processing scheme. Eventually, the IFAC institute, Florence, Italy, has won this competition. It is interesting to note that all other independently developed processors are still being maintained, advanced, and tested on MIPAS data. This allows for inter-comparisons of all level 2 algorithms, which is extremely useful for validating the ORM itself on algorithm level (see also [Laeng et al., 2013]). 


\section{ML2PP}

The ML2PP is the industrial reference implementation of the scientific core implementation ORM. The main purposes of developing this instance are to

- Critically review the algorithm,

- Implement the processor in a high level language following standard coding rules,

- Implement the framework around the scientific core with read/write routines for the input and output files as they are used in the operational ground processing unit,

- Develop a software validation plan and carry out the validation against the ORM with dedicated test procedures,

- Prepare documentation on the software, the interfaces, the validation report, etc.

The history of the ML2PP started with the development phase as early as the mid 1990's which resulted in the first validated delivery in 1998. When the first operational MIPAS data were downloaded and fed into the ML2PP, the processor converged without problems. This proved the quality of the algorithm.

The ML2PP eventually turned into an operational processor when the version V6.00 became the designated processor for offline reprocessing activities for the full mission in 2012, providing L2 data sets to the user community.

Between these milestones lie the activities related to continuous improvement of the MIPAS processing in general and the L2-processing in particular. A major upgrade of the processing-scheme became necessary after a malfunction of the MIPAS instrument in 2004, which was ultimately solved by reducing the spectral resolution in 2005. The ML2PP history is still ongoing as version 7.01 is the designated software tool for the reprocessing of all valid MIPAS L1b data into L2 products in 2014. By then, a rich collection of science and data quality improvements will have been implemented that have been elaborated by the MIPAS Quality Working Group (QWG) members and supporters while analyzing and processing MIPAS data during 10 years of service.

IPF

The IPF was operational in the ground segment, thus providing the operational level 2 data sets during the mission from 2002 to 2012. The IPF instance of the algorithm is validated against the ML2PP. Having three algorithms validated against each other has certain advantages, but this ultimately became a problem after adjusting the algorithm for the optimized MIPAS resolution, due to the instrument malfunction in 2004. The sequential adjustment from the ORM via ML2PP to the IPF actually delayed the operational ground processing for years. In order to speed up the validation phase, it was discussed to drop either the ML2PP or the IPF from the validation chain. Due to the unexpected end of mission, it never came to that decision, and the IPF remained operational in the ground segment until end of mission. For the reprocessing campaign of the complete MIPAS data set the ML2PP was chosen as operational processor.

\section{VALIDATION CONCEPT}

The software validation concept of MIPAS level 2 processing is a two-step procedure. First, the ML2PP is validated against the scientific instance ORM. Secondly, the IPF is validated against ML2PP (cf. Figure 4). Having three independently coded instances of the same algorithm lowers the probability of coding errors, which is a true benefit for such a complex algorithm that is prone to cumulative errors.

Although the same level of quality can also be reached with two instances instead of three, the choice of having a third implementation was mainly motivated by political and not scientific interest. A clear disadvantage associated with this two-step validation is the danger of having long adjustments of all software versions when algorithmic changes are made. 
The validation procedure itself is a process containing several steps, and follows different comparison criteria. In order to compare software outputs it is necessary to compare intermediate results, especially for algorithms containing so many calculations and iterations as for the ML2PP. Thus, the algorithms have been designed to write intermediate test data sets used for validation. As an example, the pressure, temperature and volume mixing ratios are being written to files after every iteration cycle for comparison of intermediate results. The driving question for the comparison of two files is the allowed margin for the numbers being compared. Ultimately, a set of margins basically defines by how much a physical process may deviate from a clear mathematical formulation.

The margins initially proposed have been determined by running the ORM algorithm on different hardware platforms and comparing the results in terms of statistical evaluations. These statistics campaigns should lead to margins of the order of the hardware precision. In practice, such a precision is impossible to reach in different implementations, e.g. different programming languages (FORTRAN vs. $\mathrm{C}++)$, different numerical precision during calculations (floating point vs. double point variables), and different implementations of sub-routines (usage of external libraries vs. self-coded routines). Moreover, the numerical precision is also affected by low-level calculations such as taking the logarithm of a number, which reduces the accuracy. Also, the sequence of adding, subtracting, dividing, and multiplying numbers can be important when very big and very small numbers are involved.

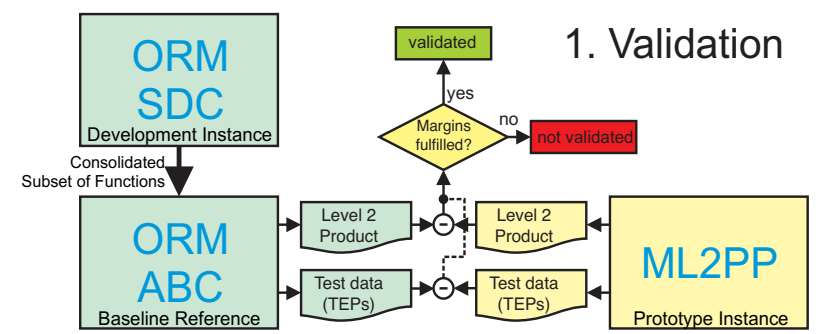

Figure 4: Validation concept
The margins certainly had to be increased taking into account these fundamental differences. An analytical approach was not feasible because of the algorithm's complexity. Especially the large numbers of iterations during the processing of several scans in sequence let errors accumulate, and thus further degrade the numerical accuracy between both algorithms. The choice for margins was then solved by comparing the intermediate ML2PP results with ORM results for a robust test case where both algorithms converged after the same number of iterations to a very similar result. Margins have been defined for all parameters written to the intermediate files and to the level 2 product file. Ultimately, these margins are much higher than the initially proposed hardware precision, but are still small enough to state that the processing results of both different algorithms can be labeled as being 'identical'.

The margins for all values of the test outputs as well as for the level 2 output have been reported in a corresponding 'Test Definition and Procedure Document'. In order to provide an example of the selected margins, some results are shown in the conclusion section. All margins and thus the precision requirements were fulfilled by ORM vs. ML2PP and ML2PP vs. IPF. Thus, the operational software is considered validated.

\section{RESULTS AND CONCLUSION}

This paper discussed the three ESA processors ORM, ML2PP and IPF for MIPAS L1b to L2 processing with focus on the ML2PP by presenting its

- General processing scheme,

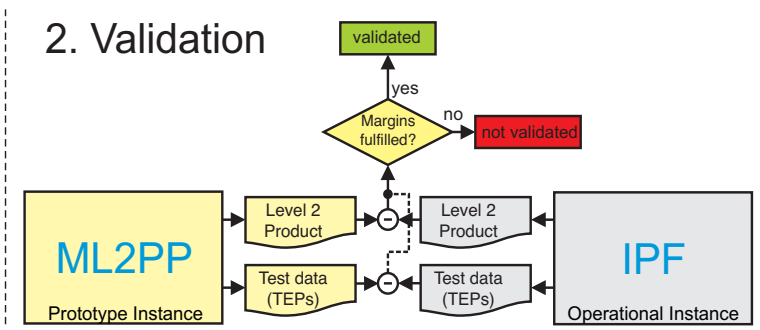



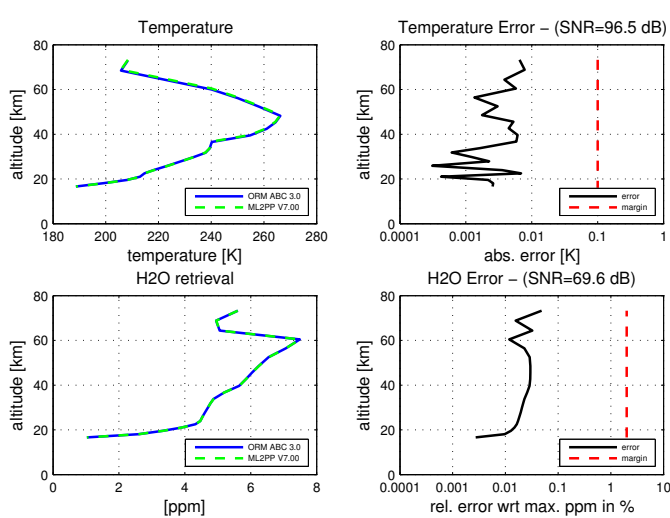

Figure 5: Comparison of processor outputs

- Role during the mission,

- Software validation concept.

The operational software validation is done by comparing ORM results with ML2PP results. For this comparison some margins were defined for deciding whether the outputs can be considered 'identical'. These comparisons are made for all values in the level 2 product and for intermediate results.

As an example, the temperature and water vapour retrieval results of the scientific processor (ORM) and of ML2PP are plotted on the left hand side in figure 5 . Both plot lines appear congruent, which is the expected result. On the right hand side, the numerical differences are plotted on a logarithmic abscissa. The allowed margins (vertical red lines) are usually percentage values of the maximum retrieval value.

For water vapour retrieval, the allowed error is two percent of the maximum value (here: maximum is around $7.5 \mathrm{ppm}$ ). The error is well below that margin by more than one magnitude, which means that a very good match of the outputs is achieved with relative errors below 0.1 percent (here: error is lower than $0.0075 \mathrm{ppm}$ ).

For temperature retrieval, two percent of the maximum would mean a margin of a few Kelvin, which is higher than the MIPAS accuracy requirement of $0.1 \mathrm{~K}$. Therefore, the plots show a stricter absolute margin of $0.1 \mathrm{~K}$. Again, the error is well below that margin with accuracy better than $0.01 \mathrm{~K}$.

As a conclusion, software implementation validation is an important component of the entire MIPAS level 2 product validation chain. The ML2PP has been performing this task for the MIPAS mission since mid 1990's. Eventually, the ML2PP was chosen to provide the operational MIPAS L2 products since 2012.

\section{REFERENCES}

[Carli et al., 2013] B. Carli, M. Carlotti, S. Ceccherini, M. Höpfner, P. Raspollini, M. Ridolfi, L. Santurri, and L. Sgheri (2013). MIPAS Level 2 Algorithm Theoretical Baseline Document, issue 6.0 http://www2.fci.unibo.it/ ridolfi/mipas/docs/at bd/atbd_issue_6.0.pdf (December 19, 2013).

[Raspollini et al., 2013] Raspollini, P., Carli, B., Carlotti, M., Ceccherini, S., Dehn, A., Dinelli, B. M., Dudhia, A., Flaud, J.-M., López-Puertas, M., Niro, F., Remedios, J. J., Ridolfi, M., Sembhi, H., Sgheri, L., and von Clarmann, T.: Ten years of MIPAS measurements with ESA Level 2 processor V6 - Part 1: Retrieval algorithm and diagnostics of the products, Atmos. Meas. Tech., 6, 2419-2439, doi:10.5194/amt-6-2419-2013, 2013.

[Laeng et al., 2013] Laeng, A, Hubert, D, Verhoelst, T, von Clarmann, T, Dinelli, B.M., Dudhia, A., Raspollini, P., Stiller, G., Grabowski, U., Keppens, A., Kiefer, M., Sofieva, V., Froideveaux, L., Walker, K.A., Lambert, J.-C., Zehner, C., The Ozone Climate Change Initiative: Comparison of four Level 2 Processors for the Michelson Interferometer for Passive Atmospheric Sounding (MIPAS), submitted to Remote Sensing of Environment, 2013. 